\title{
Estimation and prediction of weather variables from surveillance data using spatio-temporal Kriging
}

\author{
Ramon Dalmau, Marc Pérez-Batlle and Xavier Prats \\ Telecommunications and Aerospace Engineering School of Castelldefels \\ Department of Physics - Aeronautics division \\ Technical University of Catalonia \\ Castelldefels, Spain 08860
}

\begin{abstract}
State-of-the-art weather data obtained from numerical weather predictions are unlikely to satisfy the requirements of the future air traffic management system. A potential approach to improve the resolution and accuracy of the weather predictions could consist on using airborne aircraft as meteorological sensors, which would provide up-to-date weather observations to the surrounding aircraft and ground systems. This paper proposes to use Kriging, a geostatistical interpolation technique, to create shortterm weather predictions from scattered weather observations derived from surveillance data. Results show that this method can accurately capture the spatio-temporal distribution of the temperature and wind fields, allowing to obtain high-quality local, short-term weather predictions and providing at the same time a measure of the uncertainty associated with the prediction.
\end{abstract}

\section{INTRODUCTION}

In the forthcoming Trajectory Based Operations (TBO) concept, each aircraft will be represented by a four-dimensional (4D) trajectory to strategically manage and tactically control operations. In this context, the provision of updated and accurate weather data to the on-board and ground-based trajectory predictors (TPs) will be a key requirement to improve the flight predictability and, consequently, to increase the performance of the overall Air Traffic Management (ATM) system.

The accuracy of the trajectories computed by TPs, and especially the computation of the estimated time of arrival over a fix in the flight plan, critically depends on the quality of the weather data. The sensitivity of the trajectory predictions to weather uncertainties was already investigated in [1], [2].

State-of-the-art weather forecasts used in most ATM applications are based on Numerical Weather Predictions (NWP). Nowadays, the observations required to initialize NWP models are mainly gathered from radiosondes and aircraft equipped with AMDAR (Aircraft Meteorological Data Relay). However, the spatial distribution of the radiosondes (which are launched only two to four times a day) is too coarse, and aircraft observations gathered from AMDAR are not sufficient because not all aircraft in operation are equipped with such system [3].

In addition, conventional NWP models are updated at relatively long intervals, and may not have sufficient resolution to enable accurate 4D predictions. Last but not least, using such deterministic weather models makes it impossible to estimate the uncertainties involved with the predicted trajectories. Due to these limitations, state-of-the-art weather predictions are unlikely to satisfy the future ATM system requirements [4].
A potential solution to improve the resolution, frequency and quality of weather predictions for forecasting and nowcasting purposes is to use aircraft airborne as meteorological sensors [5], [6]. Several works already proposed the use of weather observations derived from secondary surveillance radar Mode $\mathrm{S}$ replies to provide enhanced wind and temperature information for the meteorological and ATM community (see for instance [7], [8] and the references therein).

In [9], local wind profiles (as a function of the altitude only) were estimated in real-time by using wind measurements gathered from nearby aircraft. The potential benefit of sharing wind measures was investigated in [10]. Having such distributed weather information system, on-board and ground-based TPs would be able to improve the short-term trajectory predictions.

Most of the previous works considered the wind as a 2D field, mainly focusing on the en-route phase of flight, where aircraft are strategically separated in flight levels [11], [8].

In addition, most of the proposed algorithms predict the weather variables at unknown locations based on straightforward interpolation methods such as nearest point, trend surface or Inverse Distance Weight (IDW), which are not able to capture the spatio-temporal correlation between observations [9], [8].

Another feature that previous methods do not possess is the capability of providing the confidence level of the predictions.

Geostatistical interpolation methods such as Kriging have been already used to create wind estimations and predictions at unknown points from scattered observations, showing superior performance than standard interpolation methods [12]. However, the potential applicability of such techniques to generate short-term weather predictions given observations derived from Mode $\mathrm{S}$ messages has not been addressed before.

The main contribution of this paper is to present the working principle of an algorithm inspired on geostatistics suitable to generate short-term weather predictions from scattered observations, which takes into account the spatio-temporal correlation between them. The proposed algorithm also provides a measure of the uncertainty associated with the prediction.

Weather observations derived from Mode S messages have been used to experimentally extract geostatistical parameters of the local wind and temperature fields. Thereafter, spatiotemporal universal Kriging (UK-ST) has been used to generate 4D wind and temperature predictions for the region of study. 


\section{BACKGROUND}

Most interpolation methods estimate the value of a variable (e.g. temperature or wind) at an unknown location as a weighted sum of observations obtained at surrounding locations. Typically, the weights are assigned according to functions that give a decreasing weight with increasing distance.

Interpolation methods may be classified into deterministic and geostatistical. The former use specific mathematical functions to assign these weights. Examples of deterministic methods are Inverse Distance Weight (IDW) and Radial Dasis Functions (RBF) [13]. The latter use data-driven statistical models that take into account correlation between data. The most representative example of these is Kriging.

Section II-A presents a methodology to derive weather variables from surveillance data. In Section II-B the Kriging algorithm and its most well known variants are described.

\section{A. Derivation of weather variables from Mode $S$ data}

The track and turn (BDS50) and the heading and speed (BDS60) Mode S messages were designed to aid conflict detection and long-term Air Traffic Control (ATC) functions in maintaining accurate aircraft horizontal track positions and velocities. It is required to combine the information contained in a BDS50 and a BDS60 message to derive meteorological data. Table I shows the flight data contained in these messages.

TABLE I

FLIGHT DATA PROVIDED BY MOdE S MESSAGES

\begin{tabular}{ccc}
\hline BDS message & Variable & Symbol \\
& Roll angle & $\phi$ \\
BDS50 & Track angle & $\chi_{g}$ \\
& Ground speed & $v_{g}$ \\
& True airspeed (TAS) & $v_{a}$ \\
& Track angle rate & $\dot{\chi}_{g}$ \\
\hline \multirow{3}{*}{ BDS60 } & Aerodynamic heading & $\chi_{a}$ \\
& Indicated airspeed (IAS) & $v_{I A S}$ \\
& Mach number & $M$ \\
& Barometric vertical rate & $\dot{h}_{p}$ \\
& Inertial vertical velocity & $\dot{h}$ \\
\hline
\end{tabular}

1) Temperature derivation: The TAS is not directly observed by the aircraft, but derived from the sensed temperature $(\tau)$ and the measured $M$ using the following relationship:

$$
v_{a}=M \sqrt{\gamma_{a} R \tau}
$$

where $a_{s}=\sqrt{\gamma_{a} R \tau}$ is the speed of sound; $\gamma_{a}$ is the specific heat ratio of the air and $R$ the specific gas constant for dry air. The inverse procedure can be performed to obtain $\tau$ from the observed $M$ and $v_{a}$ :

$$
\tau=\frac{1}{R \gamma}\left(\frac{v_{a}}{M}\right)^{2}
$$

2) Pressure derivation: Operational altitudes in aviation are always given in terms of pressure altitude (i.e. the altitude displayed by on-board barometric altimeters). This altitude only depends on the atmospheric pressure, and is computed assuming International Standard Atmosphere (ISA) and the principle of hydrostatic equilibrium. The inverse procedure can be performed to obtain the pressure $(p)$ from the observed $h_{p}$ :

$$
p= \begin{cases}p_{0}\left(\frac{\tau_{0}-\lambda_{\tau} h_{p}}{\tau_{0}}\right)^{\frac{g}{\lambda \tau R}} & \text { if } h_{p} \leq h_{11} \\ p_{11} \exp \left(\frac{-g\left(h_{p}-h_{11}\right)}{R \tau_{11}}\right) & \text { if } h_{p}>h_{11}\end{cases}
$$

where $g$ is the gravity acceleration; $\lambda_{\tau}$ is the ISA temperature lapse rate; $\tau_{0}$ and $p_{0}$ are the temperature and pressure values at sea level, respectively; and $h_{11}, \tau_{11}$ and $p_{11}$ are the altitude, temperature and pressure at the tropopause, respectively.

3) Density derivation: The IAS is the speed of an aircraft callibrated to reflect standard atmosphere adiabatic compressible flow at sea level. The following formula relates the IAS with the TAS, $p$, the density $(\rho)$ and $M$ :

$$
v_{I A S}=v_{a} f(p, M) \sqrt{\frac{\rho}{\rho_{0}}}
$$

where the function $f$ is given by[3]:

$f(p, M)=1+\frac{1}{8}\left(1-\frac{p}{p_{0}}\right) M^{2}+\frac{3}{640}\left(1-10 \frac{p}{p_{0}}+9 \frac{p^{2}}{p_{0}^{2}}\right) M^{4}$

The density of the air can be deducted by combining Eqs. (4) and (5) and solving for $\rho$ :

$$
\rho=\rho_{0}\left(\frac{v_{I A S}}{f(p, M) v_{a}}\right)^{2}
$$

where $M, v_{a}$ and $v_{I A S}$ are directly obtained from the Mode $\mathrm{S}$ messages, and $p$ is computed by using Eq. (3).

4) Wind vector derivation: The wind vector $\left(\boldsymbol{V}_{w}=\left\langle w, \not \chi_{w}\right\rangle\right)$ can be obtained as the difference between the track vector $\left(\boldsymbol{V}_{g}=\left\langle v_{g},\left\langle\chi_{g}\right\rangle\right)\right.$ and the heading vector $\left(\boldsymbol{V}_{a}=\left\langle v_{a}, \underline{\chi_{a}}\right\rangle\right)$ :

$$
\boldsymbol{V}_{w}=\boldsymbol{V}_{g}-\boldsymbol{V}_{a}
$$

Equation (7) is valid for the cruise phase (flying at constant altitude) and could be also appropriate during the descent, where the flight path angle is relatively small. During climb, however, the flight path angle cannot be neglected, and the following expression should be used for $\boldsymbol{V}_{a}$ :

$$
\boldsymbol{V}_{a}=\left\langle v_{a} \sqrt{1-\left(\dot{h} / v_{a}\right)^{2}},\left\langle\chi_{a}\right\rangle\right.
$$

The north and east wind components $\left(w_{n}\right.$ and $w_{e}$, respectively) can be computed from $\boldsymbol{V}_{w}$ using elemental trigonometric properties. 


\section{B. Kriging interpolation}

The goal of the Kriging interpolation algorithm is to provide the best estimate of a variable $Z$ at an unmeasured location $\boldsymbol{x}$ from a set of surrounding data points, where $\boldsymbol{x}$ is the $n$ dimensional coordinate vector. $Z$ is treated as a random field, composed by a deterministic trend $(m)$ and a residual $(\epsilon)$ :

$$
Z(\boldsymbol{x})-m(\boldsymbol{x})=\epsilon(\boldsymbol{x})
$$

All the Kriging variants are based on the following linear regression estimator $Z^{*}(\boldsymbol{x})$, which estimates the residual at $\boldsymbol{x}$ as a weighted sum of residuals at surrounding data points:

$$
Z^{*}(\boldsymbol{x})-m(\boldsymbol{x})=\sum_{\boldsymbol{y} \in \mathcal{X}} \lambda_{\boldsymbol{y}}(Z(\boldsymbol{y})-m(\boldsymbol{y}))
$$

where $\mathcal{X}$ is the set of points in the local neighborhood of $x$ used for the estimation of $Z(\boldsymbol{x})$; and $\lambda_{\boldsymbol{y}}$ is the Kriging weight assigned to the surrounding data point $\boldsymbol{y} \in \mathcal{X}$.

The weights $\lambda \boldsymbol{y}$ are obtained by minimizing the variance of the estimator $\left(\sigma^{2}\right)$ :

$$
\sigma^{2}(\boldsymbol{x})=\operatorname{Var}\left\{Z^{*}(\boldsymbol{x})-Z(\boldsymbol{x})\right\}
$$

subject to the following unbiasedness constraint:

$$
\mathrm{E}\left\{Z^{*}(\boldsymbol{x})-Z(\boldsymbol{x})\right\}=0
$$

The residual component is treated as a zero-mean stationary random variable:

$$
\begin{aligned}
\mathrm{E}\{\epsilon(\boldsymbol{x})\} & =0 \\
\operatorname{Cov}\{\epsilon(\boldsymbol{x}), \epsilon(\boldsymbol{x}+d)\} & =C_{\epsilon}(d)
\end{aligned}
$$

where the stationary covariance function $C_{\epsilon}$ reflects the covariance between any pair of residuals separated by an Euclidean distance $d$, independently of the position of $\boldsymbol{x}$ in the domain.

The residual covariance function is obtained from a semivariogram model $(\gamma)$ that quantifies the spatial correlation between data at different sample locations:

$$
C_{\epsilon}(d)=C_{\epsilon}(0)-\gamma(d) .
$$

The semivariogram is computed experimentally by applying the following formula to whole set of known data points:

$$
\gamma(d)=\frac{1}{2 N(d)} \sum_{N(d)}(Z(\boldsymbol{x})-Z(\boldsymbol{x}+d))^{2}
$$

where $N(d)$ is the number of pairs of data points with distance $d$ apart. It should be noted that the semivariance may be configured to account for direction-dependent spatial variability (also known as anisotropy). In addition, for spatiotemporal Kriging, $\gamma$ is also a function of the time lag.

Each physical phenomenon has its own experimental semivariogram, which ideally can be fitted by an appropriate mathematical function. There exist a wide variety of semivariogram models, including spherical, gaussian, exponential and linear.

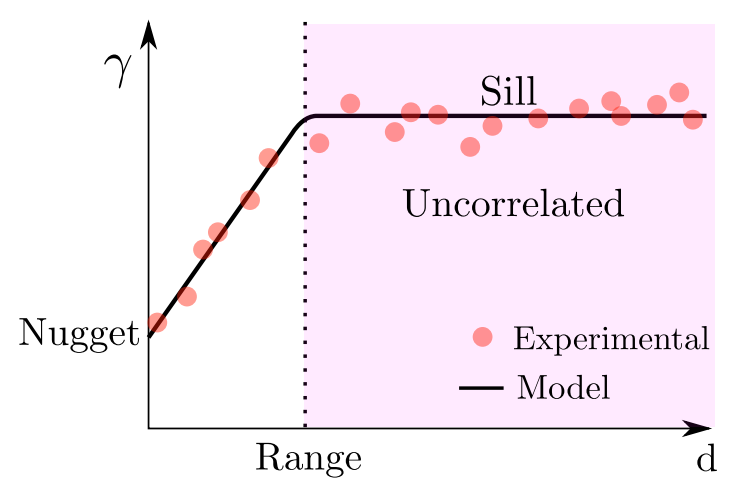

Fig. 1. Typical spherical variogram

Figure 1 shows a typical spherical semivariogram model fitting hypothetical experimental data.

In most phenomenon found in the nature, the difference in values for two locations close to each-other tend to be small (i.e. low semivariance). In the extreme case, two points with equal coordinates are expected to have exactly the same value. In practice, however, randomness and measurement errors lead to what is called a nugget effect, indicating that the variable subject of study changes abruptly at a very small scale.

As the distance between locations increase, the data points being compared one to each-other are less likely to be similar (i.e. the semivariance is large). There exists a distance called range from which the semivariogram asymptotically converges to a constant value. The range represents the maximum distance beyond which measurements are no longer correlated. The sill is the value at which the semivariogram appears to level off, and represents the semivariance of the random variable at large distances, when data points become uncorrelated.

The different variants of Kriging are distinguished according to the trend model. For instance, simple Kriging (SK) assumes $m$ as a known and constant scalar $m_{S K}$ all over the study area (i.e. $\left.m(\boldsymbol{x})=m_{S K}\right)$. In ordinary Kriging (OK), rather than assuming a constant mean over the entire domain, its value is assumed to be constant only in the local neighborhood of each estimation point. As a matter of fact, OK is a zero-order case of a more generic variant called universal Kriging (UK). In UK, $m(\boldsymbol{x})$ is fitted as a smooth function of the coordinate vector $\boldsymbol{x}$ and is represented by a polynomial. For instance, a first-order trend model would be given by:

$$
m(\boldsymbol{x})=a_{0}+\sum_{i=1}^{n} a_{i} x_{i}
$$

where $a_{i}(i \in\{1,2, \ldots, n\})$ are coefficients obtained from a linear regression performed with the data points in $\mathcal{X}$.

It should be noted that in UK the trend is restricted to be a function of the spatial coordinates. If the trend is defined externally, as a function of secondary variables, the term Kriging with external drift (KED) is preferably used [14].

Another widely used variant is the regression Kriging (RK), which is accomplished in two steps. First, $m$ is modeled as a function of the spatial coordinates and/or secondary variables. 
This is performed as an independently process using linear regression techniques with the whole set of data points (not only those in the neighborhood of $\boldsymbol{x}$ ). Once the deterministic part of the random variable has been estimated, the residual are interpolated using $\mathrm{OK}$ and are added back to $\mathrm{m}$.

The Kriging method described by Eq. (10) applies to both purely spatial and spatio-temporal data. Although the extension to spatio-temporal geostatistics is not trivial. On the one hand, spatial and temporal domains have different scales, causality principles and variability patterns, which lead to significant challenges when trying to capture spatio-temporal correlations. On the other hand, while predictions in space are basically interpolations, time predictions are extrapolations.

Two main groups of spatio-temporal variogram models exist: separable, composed by a combination of purely spatial and purely temporal semivariograms; and non-separable, which are more flexible to handle empirical covariances showed up in "real world" data. A widely used non-separable model is the sum-metric model, which assumes that the residuals $\epsilon$ consist of three stationary and independent components:

$$
\gamma(d, \Delta)=\gamma_{s}(d)+\gamma_{t}(\Delta)+\gamma_{s t}\left(\sqrt{d^{2}+(\alpha \Delta)^{2}}\right)
$$

where $\gamma(d, \Delta)$ is the semivariance of variable $Z(\boldsymbol{x}, t)$ for distance $d$ in space and lag $\Delta$ in time; $\gamma_{s}$ and $\gamma_{t}$ are spatial and temporal semivariogram components respectively, each one with its particular parameters; and $\gamma_{s t}$ is the joint spatio-temporal component. Besides the typical semivariogram paramters (sill, nugget and range), $\gamma_{s t}$ also includes a parameter $(\alpha)$ for the conversion of temporal lag to spatial distance.

\section{SPATIO-TEMPORAL UNIVERSAL KRIGING SET-UP}

In this paper, the proposed method is illustrated for the problem of predicting the temperature and the wind, although its extension to the pressure and density should be trivial.

Section III-A shows the method adopted to collect and process the surveillance data used to derive the weather observations. Section III-B presents the trend models considered for the temperature and the two wind components $\left(w_{e}\right.$ and $\left.w_{n}\right)$.

\section{A. Surveillance data collection and processing}

The flight data from which the weather observations rely on are produced as a response to an ATC radar interrogation. In order to conserve bandwidth, the Mode S message does not provide information about the type of data contained on it.

As a consequence, the inference of the message type must be performed by the ground receiver using certain criteria. In addition, errors in the transmission of the messages could results in values with no physical sense (e.g. negative Mach number or unrealistic airspeeds). Ref. [15] presents a methodology appropriate to monitor the validity of Mode $\mathrm{S}$ messages.

In addition, during turning maneuvers the airflow around the pitot-tube can be irregular, leading to erroneous measurements. For this reason, observations with a large bank angle $(\phi)$ have been omitted in this study. The higher the maximum allowed bank angle $\left(\phi_{\max }\right)$, the more weather observations would be collected, but the lower the quality of the measurements.

The values of the different weather variables obtained by using the methodology presented in Section II-A must be associated with a point in space and time. Mode S-derived weather observations could be complemented with the aircraft position as observed by the tracking radar. This approach, however, requires to have access to the tracking radar position data, which unfortunately is not always possible.

A different solution consists on inferring the position where a given Mode $\mathrm{S}$ messages was sent taking advantage of free to air Automatic Dependent Surveillance-Broadcast (ADS-B) position reports emitted by the corresponding aircraft at the a near time instant. The advantage of this method (apart from not requiring access to the tracking radar) is that the quality of the position data obtained from ADS-B is expected to be higher than those provided by the tracking radar.

A weather observation is considered valid if and only if an ADS-B position report from the same aircraft is received in a near time instant. Otherwise, the weather observation is discarded. The larger the maximum time allowed between a weather observation and a position report $\left(\Delta t_{\max }\right)$, the higher the volume of weather observations that would be gathered, but the lower the confidence on their associated positions.

Last but not least, the latitude and longitude data contained in the ADS-B position report are expressed in decimal datum, while the GPS altitude is given in meters. The coordinates obtained from ADS-B need to be converted from WGS84 into Universal Transverse Mercator (UTM) coordinate system to allow for computation of distances and angles between data points using Euclidean geometry during the Kriging process.

In this paper, the coordinates vector associated to each data point $\boldsymbol{x}=[n, e, h]$ is composed, respectively, by the north position and the east position in UTM, and the GPS altitude. All these coordinates must be expressed in the same units.

\section{B. Trends modeling}

In this paper the spatio-temporal UK (UK-ST) variant has been selected, which assumes that the trend $m$ is a function of the spatial coordinates. Section III-B1 shows the model adopted for the temperature trend, while Section III-B2 shows the trend model for the wind speed.

1) Temperature trend model: It is well known that the relationship between air temperature and altitude is approximately linear in the troposhere, while in the tropopause it remains constant up to the stratosphere (well above the altitudes where commercial aircraft operate). Therefore, it is enticing to formulate the trend model for the temperature as:

$$
m(\boldsymbol{x})=a_{0}-a_{1} h
$$

where the coefficients $a_{0}$ and $a_{1}$ are obtained during the Kriging process itself. For each $\boldsymbol{x}$, these coefficients are obtained by performing a linear regression with all the temperature data in $\mathcal{X}$. For this reason, this trend model would give excellent performances both in the troposphere and the tropopause. 
2) Wind components trend model: The wind speed (both north and east components) trend is constructed based on the following standard power-law wind profile:

$$
w_{k}(\boldsymbol{x})=W_{k}\left(\frac{h}{H_{k}}\right)^{\alpha_{k}} ; \quad k \in\{n, e\}
$$

where $W_{k}$ is a known wind speed at $H_{k} ; \alpha_{k}$ is the Hellman exponent, which depends on many factors, such as the coastal location, the shape of the terrain on the ground, and the stability of the air. The typical value for neutral stability conditions is $1 / 7$. Taking logarithms in both sides of Eq. (7) the following trend for the transformed wind speed is obtained:

$$
m(\boldsymbol{x})=a_{0}+a_{1} \log h
$$

It should be noted that all the computation of the semivariongrams and Kriging weights will be performed on the transformed data $\log w_{k}, k \in\{n, e\}$. Then, the outcome of the Kriging interpolation should be transformed back. While transforming back the wind speed itself is straightforward, one must be cautious when transforming back its variance.

\section{RESULTS}

In this paper, the generic UK-ST algorithm presented in Section II-B has been particularized with the trend models presented in Section III-B. The UK-ST framework as implemented in the gstat package (in R) [16] has been used to perform the spatio-temporal predictions for the temperature and wind speed components over the region of interest.

Section IV-A presents the set of surveillance data used for this study. Section IV-B shows the experimental and modeled semivariograms for the temperature and wind speed. Finally, Sections IV-C and IV-D assess the performance of the algorithm by means of cross-validation and by predicting the weather along a real descent trajectory, and comparing the predictions with the observations obtained from that flight.

\section{A. Data set}

Surveillance data collected during 24 hours (starting the $1^{\text {st }}$ January 2017 at 17:00) with a receiver and an antenna located nearby Barcelona-El Prat airport (Spain) has been used for this experiment. These data included both Mode S and ADS$B$ messages, which were processed following the methodology presented in Section III-A. For this experiment, $\phi_{\max }$ and $\Delta t_{\max }$ have been set to $5^{\circ}$ and 1 second, respectively.

As a result, a set of 36,190 weather observations have been obtained, each one associated with a 4D point (north and east coordinates, altitude and time) and containing information about temperature, pressure, density and wind vector.

Not all the whole set of data has been used in this study. A random sample of 6,000 weather observations between 3,000 ft and FL350 has been selected, aiming to reduce the computational burden of the semivariogram computation, which can take up to 30 minutes to complete for each variable.

Figure 2 shows the weather observations from this sample set in UTM coordinate system. This Figure also shows the descending trajectory chosen to assess the performance of the proposed algorithm when extrapolating in time.

The directional antenna used to gather surveillance data is heading to $20^{\circ}$, with an horizontal beamwidth of $40^{\circ}$. This fact can be observed in Fig. 2, where the great majority of weather observations are located at the north-east region of the airport. Nevertheless, certain scatter observations sent from other theoretically unobserved locations have been obtained as a result of signal rebounds and the multi-path phenomenon.

The temperature and wind profiles as a function of the altitude are shown in Fig. 3. As expected, the temperature shows a linear relationship with respect to the altitude, while the wind profile is more difficult to fit with a simple mathematical function. For the particular time interval considered in this paper, the wind significantly changed its direction during the night. This event can be observed in Fig. 3(b), where the north wind component shows two well differentiated regions, each one corresponding to a different moment of the day.

\section{B. Spatio-temporal variograms}

The experimental spatio-tempiral variograms for the temperature and wind speeds have been computed by using an extension of Eq. (15) accounting for both time and space variability. Then, they have been fitted by using the summetric spatio-temporal variogram described by Eq. (17). In both cases, the temporal, spatial and joint semivariograms have been modelled as spherical functions, whose parameters (nugget, sill and range) have been determined by using a leastsquare fitting with the experimental semivariogram.

Figure 4 show the sample (or experminetal) and modelled spatio-temporal sum-metric variograms for the weather variables subject of study. The maximum distance in this Figure has been set to $100 \mathrm{Km}$, while the maximum time is 6 hours.

According to Fig. 4(a) the temperature semivariance presents a clear spherical behavior with respect to the spatial coordinate, while the dependence with respect to time is not that significant, specially at large distances.

For the north and east wind components, the semivariance shows also a clear spherical behavior in the space domain, and idefinitely increases with time for the time range considered.

\section{Cross-validation}

Various methods of varying complexity are available to assess the accuracy of a predictive model. Principal of them in geostatistics is the leave-one-out cross-validation (also known as jackknifing), which gives a clue of how well the model will perform in practice predicting values at unknown locations. The statistical parameters resulting from this process serve as diagnostics that indicate whether the model and its associated parameters (i.e. searching neighborhood and semivariogram function) are representative of the real phenomenon.

A virtue of jackknifing is that no reserved data is required to assess the performance the model. Instead, each data point is systematically suppressed from the dataset, and then predicted based on the Kriging model using the remaining data. Finally, 


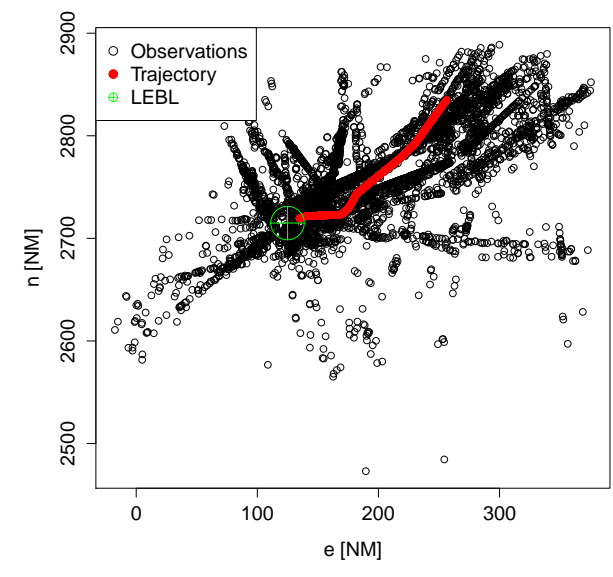

(a) $2 \mathrm{D}$ view

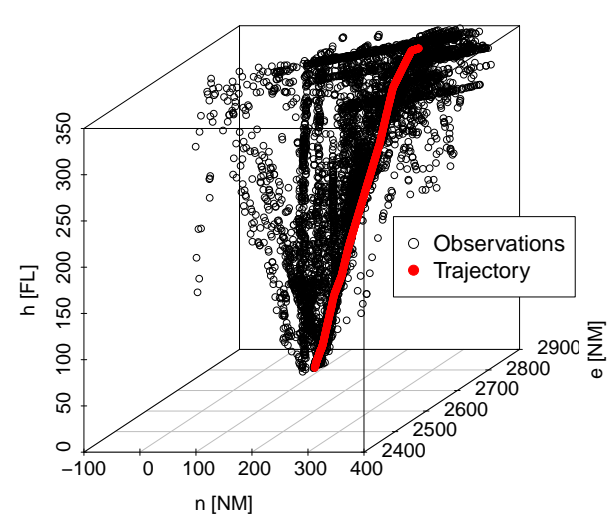

(b) $3 \mathrm{D}$ view

Fig. 2. Weather observations

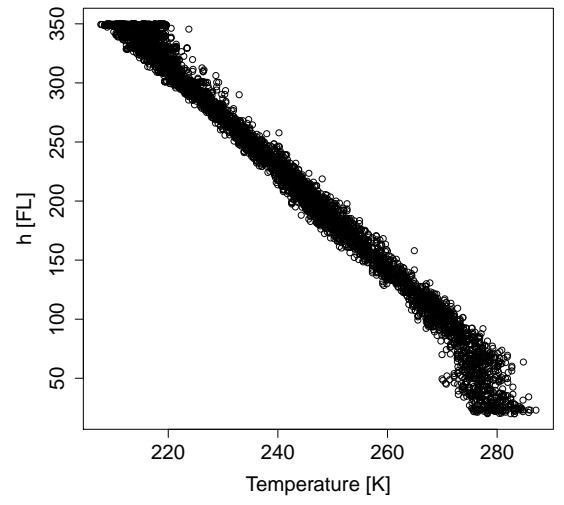

(a) Temperature

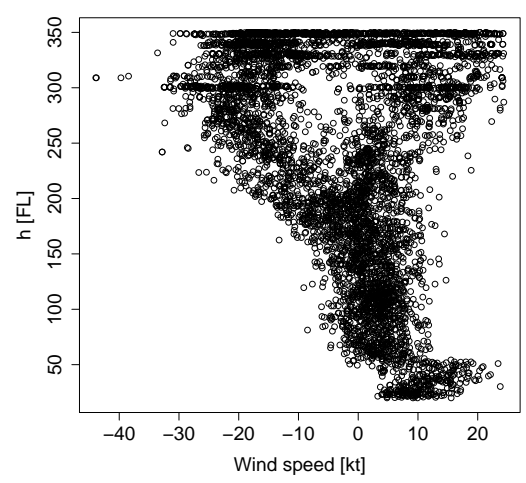

(b) North wind

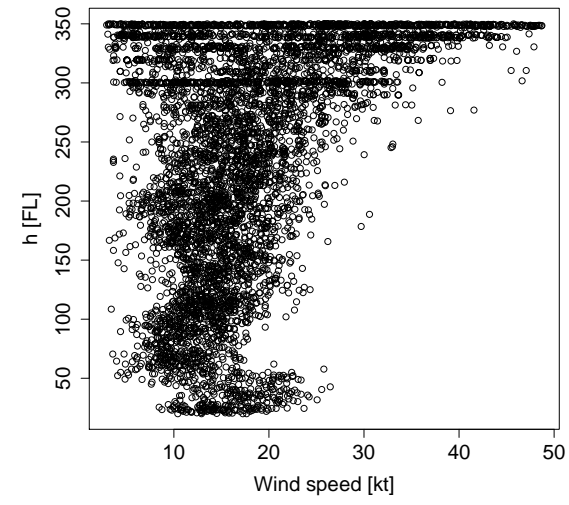

(c) East wind

Fig. 3. Weather observations

predicted and observed values are compared and statistical parameters are generated for the prediction errors.

Figure 5 shows the scatter plots of weather predictions versus observations, as a result of the jackknifing process.

The black lines of this Figure correspond to the regression lines that fit the scattered points. A perfect fit would have a slope of 1 , and this best-fit line would coincide with the 45degree line on the graph. I has been found that the slope of the best-fit line for the temperature and east wind is sightly higher than 1 for all the analyzed cases; that is, Kriging tends to over-predict large values and under-predict lower values. The complementary case has been observed for the north wind.

Points inside the region delimited by the two red-dashed lines comply with the requirement considered to achieve accurate trajectory predictions $(2.5 \mathrm{~K}$ for temperature and 5 $\mathrm{kt}$ for wind speed). The number of points not overtaking these thresholds is also shown in Fig. 5 for sake of completeness.

The leave- $N$-out cross-validation variant of jackknifing is a more stressful test based on the same rationale: a group of
$N$ data points are randomly removed from the dataset, then their associated values are predicted using the remaining data. Finally, the predicted and observed values at the location of the omitted point are compared. This process is repeated on all possible ways to cut the original dataset on a group of $N$.

Table II shows the prediction errors statistics as a result of the leave-one-out and the leave-250-out cross-validation processes, using the model presented in Section III-B and the dataset described in Section IV-A. For each weather variable and cross-validation variant the root-mean square error (RMSE), the mean absolute error (MAE), the mean error (ME), and the correlation coefficient (COR) between observations and predictions are shown.

According to Table II the RMSE for both temperature and wind speed components satisfy the requirements of the future ATM system based on time control. It is interesting to note that, for all the weather variables analyzed in this paper, Kriging tends to sightly underestimate the observed values.

The correlation coefficients shown in Table II are very close 


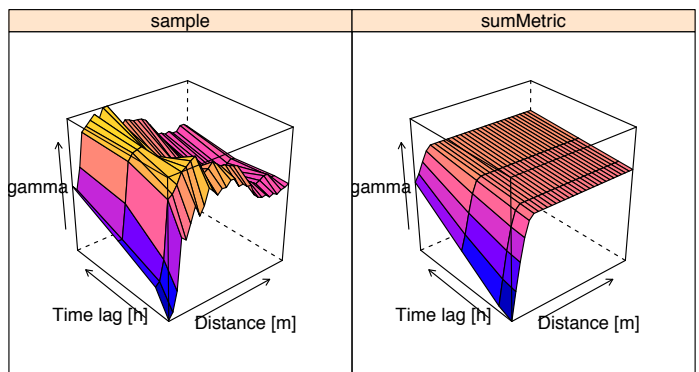

(a) Temperature

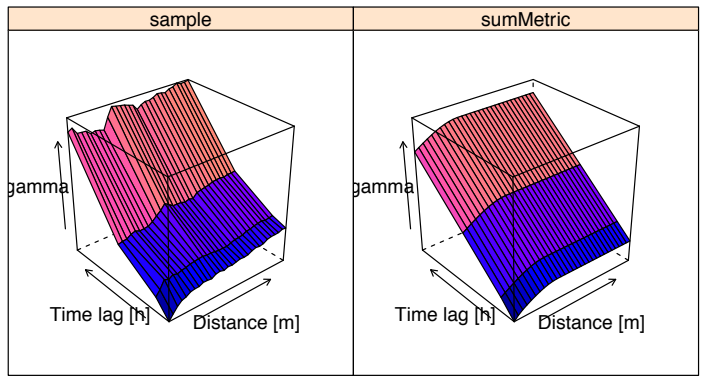

(b) North wind

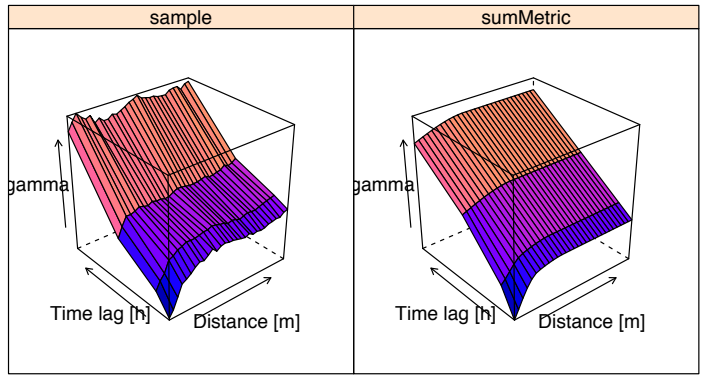

(c) East wind

Fig. 4. Spatio-temporal variograms

TABLE II

CROSS-VALIDATION ANALYSIS RESULTS

\begin{tabular}{lccccc}
\hline \multirow{2}{*}{ Variable } & \multicolumn{5}{c}{ Parameter } \\
\cline { 2 - 6 } & $N$ & RMSE & MEA & ME & COR \\
\hline \multirow{2}{*}{ Temperature } & 1 & $1.7125 \mathrm{~K}$ & $1.2994 \mathrm{~K}$ & $0.0109 \mathrm{~K}$ & 0.9969 \\
& 250 & $1.7228 \mathrm{~K}$ & $1.3061 \mathrm{~K}$ & $0.0101 \mathrm{~K}$ & 0.9969 \\
\cline { 2 - 6 } North wind & 1 & $3.5633 \mathrm{kt}$ & $2.3499 \mathrm{kt}$ & $0.1735 \mathrm{kt}$ & 0.9568 \\
& 250 & $3.5830 \mathrm{kt}$ & $2.3825 \mathrm{kt}$ & $0.1830 \mathrm{kt}$ & 0.9564 \\
\cline { 2 - 6 } East wind & 1 & $3.1024 \mathrm{kt}$ & $2.2305 \mathrm{kt}$ & $0.3056 \mathrm{kt}$ & 0.9356 \\
& 250 & $3.1495 \mathrm{kt}$ & $2.2622 \mathrm{kt}$ & $0.3092 \mathrm{kt}$ & 0.9335 \\
\hline
\end{tabular}

to 1 for all the variable subject of study, which indicates a strong linear relationship between the predictions and observations. Finally, it is important to remark that the performance of the algorithm almost no deteriorated when suppressing 250 points (4\%) of the observations, demonstrating its robustness.

Results shown in this Section demonstrate that the proposed algorithm would have excellent performance when predicting temperature and wind values at unknown locations and times, representing with high accuracy the spatio-temporal phenomenon being analyzed.

\section{Weather prediction along a particular trajectory}

The antenna started receiving data from the trajectory chosen for this assessment at 2017-01-02 10:31:53. It has been assumed that at that moment, the aircraft is provided with a short-term weather prediction generated with Kriging for the remaining descent, aiming to compute an accurate plan using close and fresh weather observations.

The Kriging data points used for the prediction correspond only to these weather observations gathered before computing the descent plan, while the spatio-temporal semivariograms used for the prediction are those shown in Fig. 4.

Figure 6(a) shows the predicted and the observed temperature along the descent trajectory subject of study, while Figs. 6(b) and 6(c) show the analogous results for the north and east wind speed components, respectively.

In Fig. 6, the lines extending vertically from the predictions indicate the standard deviations provided by the Kriging.

According to Fig. 6(a) the temperature predictions tend to overestimate the real values. This observation is in accordance with the results obtained from cross-validation. According to Figs. 6(b) and 6(c), north and east wind components predictions with a look-ahead time up to 23 minutes are able to accurately represent the real wind behavior, and foretell sudden changes in the wind speed and direction. Most of the points lie within the region delimited by the standard deviation of the predictions, except for few east wind observations obtained 700 seconds after receiving the fist message.

\section{Conclusions}

This paper assessed the potential applicability of Kriging, an algorithm inspired on geostatistics, to generate shortterm weather predictions from scattered weather observations derived from surveillance data. The motivation behind this approach is that the volume, frequency and coverage of weather observations collected in this way are much larger than those provided by radiosondes and AMDAR (Aircraft Meteorological Data Relay). In addition, the technology required is already in place, not requiring further investments.

Results from cross-validation show that wind and temperature models generated using this technique can accurately capture the spatio-temporal distribution of these weather variables. In addition, the performance of the algorithm to extrapolate in time (i.e. perform predictions) has been assessed by predicting the future wind and temperature along the descent trajectory of a real flight. Results show that Kriging predictions may satisfy the requirements of the future Air Traffic Management (ATM) system, providing at the same time a measure of the uncertainty. This valuable information could be used by on-board trajectory predictors to generate a set of preferred trajectories, or by ground systems to determine separation criteria appropriate for the uncertainty of the prediction [8].

A limitation of this method is that observations gathered by using aircraft as meteorological sensors are only available at regions where aircraft operate. Therefore, the applicability of Kriging over the ocean still deserves further research. 


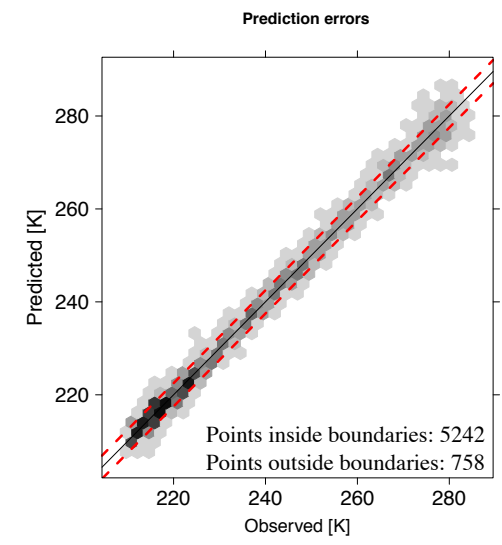

(a) Temperature

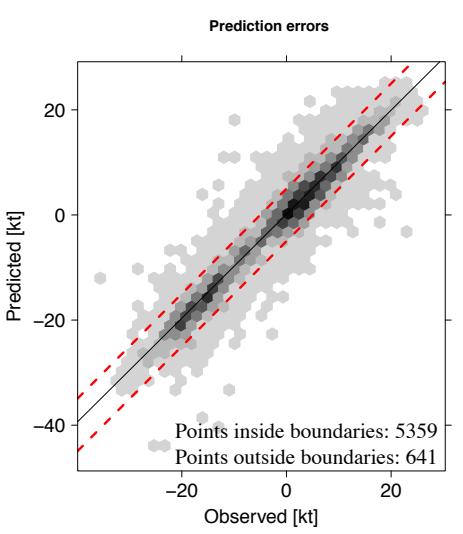

(b) North wind

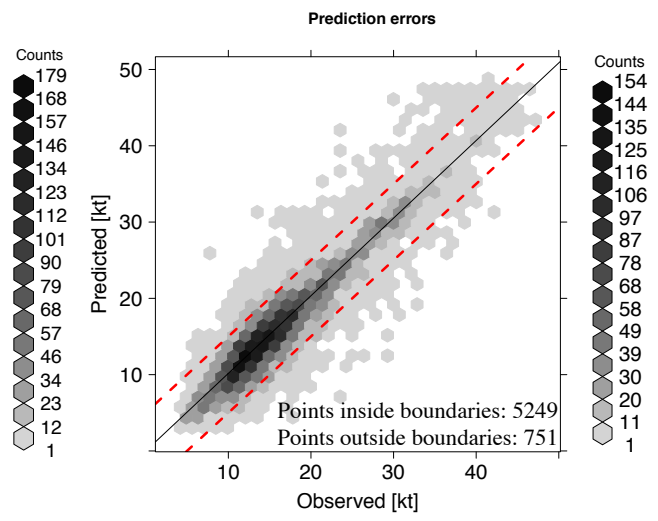

(c) East wind

Fig. 5. 1-fold cross-validation

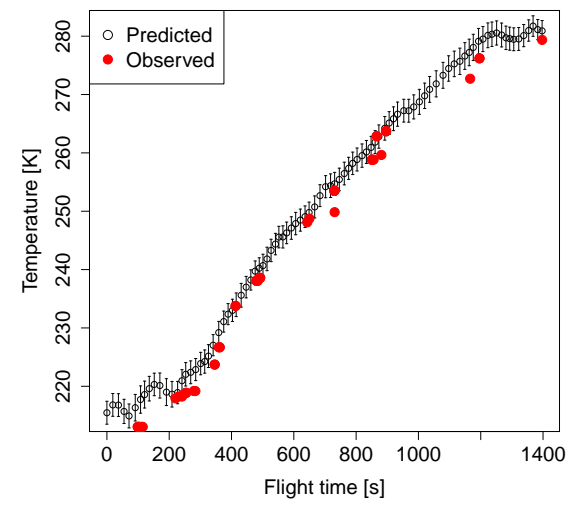

(a) Temperature

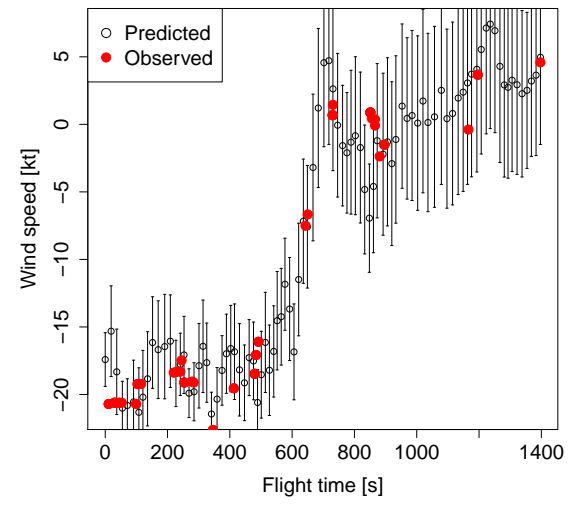

(b) North wind

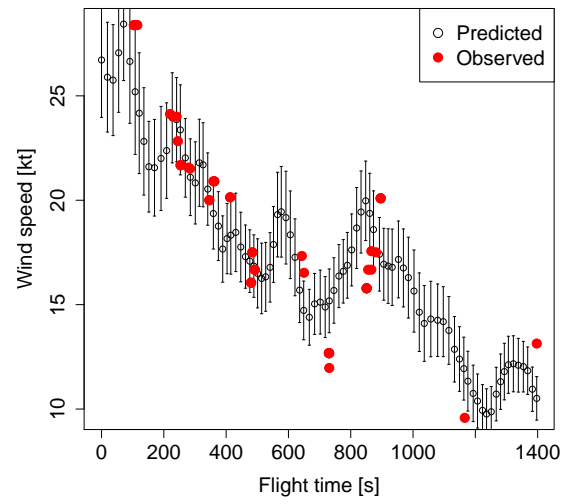

(c) East wind

Fig. 6. Kriging prediction along a trajectory

\section{REFERENCES}

[1] D. Forester and I. Dharssi, "The improvement of meteorological data for air traffic management purposes - stage 1," United Kingdom Meteorological Office, Bracknell, United Kingdom, Tech. Rep., 1992.

[2] M. R. Jackson, Z. J. Yiyuan, and S. Rhonda A, "Sensitivity of Trajectory Prediction in Air Traf c Management," Journal of Guidance, Control, and Dynamics, vol. 22, no. 2, pp. 219-228, 1999.

[3] S. D. Haan, "Quality assessment of high resolution wind and temperature observation from ModeS," The Royal Netherlands Meteorological Institute (KNMI), The Netherlands, Tech. Rep., 2010.

[4] E. Robert and D. D. Smedt, "Comparison of operational wind forecasts with recorded flight data," in ATM R\&D Seminar (ATM 2013), vol. 10, 2013.

[5] M. Hrastovec and F. Solina, "Obtaining meteorological data from aircraft with mode-S radars," IEEE Aerospace and Electronic Systems Magazine, vol. 28 , no. 12, pp. 12-24, 2013.

[6] B. Strajnar, "Validation of Mode-S Meteorological Routine Air Report aircraft observations," Journal of Geophysical Research Atmospheres, vol. 117, no. 23, pp. 1-10, 2012.

[7] J. Sondij and S. de Haan, "Aircraft as a meteorological sensor Using Mode-S Enhanced Surveillance data to derive," The Royal Netherlands Meteorological Institute (KNMI), De Bilt, The Netherlands, Tech. Rep., 2013.

[8] L. Hernando, R. M. Arnaldo, and F. J. Saez-Nieto, "Using aircraft as wind sensors for estimating accurate wind fields for air traffic management applications," Proceedings of the Institution of Mechanical Engineers , Part G : Journal of Aerospace Engineering, 2014.
[9] P. M. A. de Jong, J. J. van der Laan, A. C. Veld, M. M. van Paassen, and M. Mulder, "Wind-Profile Estimation Using Airborne Sensors," Journal of Aircraft, vol. 51, no. 6, pp. 1852-1863, 2014.

[10] K. Legrand, C. Rabut, and D. Delahaye, "Wind networking applied to aircraft trajectory prediction," in 34th IEEE/AIAA Digital Avionics Systems Conference (DASC), Sacramento, USA, 2015.

[11] D. Delahaye and S. Puechmorel, "TAS and wind estimation from radar data," in 28th AIAA/IEEE Digital Avionics Systems Conference Proceedings, Orlando, United States, 2009, pp. 2.B.5-1 -2.B.5-16.

[12] C. J. Friedland, T. A. Joyner, C. Massarra, R. V. Rohli, A. M. Treviño, S. Ghosh, C. Huyck, and M. Weatherhead, "Isotropic and anisotropic kriging approaches for interpolating surface-level wind speeds across large, geographically diverse regions," Geomatics, Natural Hazards and Risk, vol. 5705, no. July, pp. 1-18, 2016.

[13] J. Costa, L. Pronzato, and E. Thierry, "A comparison between kriging and radial basis function networks for nonlinear prediction," Laboratoire I3S, CNRS-UNSA, Biot, France, Tech. Rep., 2000.

[14] G. Hudson and H. Wackernagel, "Mapping temperature using kriging with external drift: Theory and an example from scotland," International Journal of Climatology, vol. 14, no. 1, pp. 77-91, 1994.

[15] R. D. Grappel, G. S. Harris, M. J. Kozar, and R. T. Wiken, "Elementary Surveillance (ELS) and Enhanced Surveillance (EHS) Validation via Mode S Secondary Radar Surveillance," Massachusetts Institute of Technology, Lexington, Massachusets, Tech. Rep. April, 2008.

[16] B. Grler, E. Pebesma, and G. Heuvelink, "Spatio-temporal interpolation using gstat," The R Journal, vol. 8, pp. 204-218, 2016. 